\title{
Computer simulated fragmentation arena test for assessing lethality and safety separation distances of explosive fragmentation ammunitions
}

\author{
V. M. Gold, E. L. Baker \& J. M. Pincay \\ U.S. Army RDEC-ARDEC, AMSRD-AAR-AEE-W, Picatinny Arsenal, \\ NJ, USA
}

\begin{abstract}
A combined analytical and experimental technique for determining explosive fragmentation ammunition safe separation distance and lethality without costly arena fragmentation tests is presented. The new methodology integrates high-strain high-strain-rate computer modelling with semi-empirical analytical fragmentation modelling and experimentation, offering warhead designers and ammunition developers more ammunition performance information for less money spent. The new method provides more detailed and accurate warhead fragmentation data for ammunition safe separation distance analysis, as compared to the traditional fragmentation arena testing approach.

Keywords: fragmentation modelling, fragmentation arena test, lethality, safety separation distance.
\end{abstract}

\section{Introduction}

This paper reports on a combined analytical and experimental technique for determining explosive fragmentation ammunition safe separation distances and lethality without costly arena fragmentation tests, offering warhead designers and ammunition developers more ammunition performance information for less money spent. Since the main objective of the developed procedure is to simulate the "data output" (i.e. warhead fragmentation performance information) from traditional fragmentation arena tests, a brief description of the fragmentation arena test methodology is given for completeness. 
Basic principles and requirements governing fragmentation arena test procedures in the U.S.A. are stipulated in Joint Munition Effectiveness Manual [1]. In a typical fragmentation arena test set-up, tested munitions are positioned at the origin of the reference polar coordinate system and surrounded with series of velocity-measuring screens and fragment-catching witness panels, all at significant distances from the warhead. Defining the longitudinal axis of the munition as the polar axis $z$, the polar altitudinal angles $\Theta$ are measured from the munition's nose $\left(\Theta=0^{\circ}\right)$ to the tail $\left(\Theta=180^{\circ}\right)$, and the azimuthal angles $\phi$ are measured from an arbitrary projectile's feature $\left(\phi=0^{\circ}\right)$ in a counterclockwise direction. In conventional fragmentation arena test procedures fragment sampling and fragment velocity measuring is usually limited to relatively small azimuthal sections, mainly because of enormous construction and data assessment costs associated with recovering fragments from the entire fragmenting shell. This sampling technique requires the assumption of isotropic fragmentation properties for all azimuthal angles $\phi$ throughout the entire $\Theta$-angle zone (i.e. a complete altitudinal region bounded by two polar angles). By sampling small azimuthal angles across all polar zones from the munition nose to tail and adjusting this sample data mathematically, a prediction for entire munition fragment characterization is obtained. Since only a small azimuthal section of the region is sampled and scaled up, even relatively small munition positioning errors may result in large deviations of the fragmentation data, routinely requiring repeated testing for statistical data stability.

In this work, the assessment of the ammunition fragmentation parameters was performed analytically employing the PAFRAG (Picatinny Arsenal Fragmentation) code which links three-dimensional axial symmetric high-strain high-strain-rate continuum analyses with a phenomenological fragmentation model validated through a series of experiments including flash radiography, Celotex ${ }^{\mathrm{TM}}$ and water test rear fragments recovery, and sawdust total fragment spray recovery.

In fragmentation arena tests, the ammunition fragmentation characteristics are assessed as functions of polar angles $\Theta$ identifying angular positions of fragment-catching witness panels and velocity-measuring screens. In PAFRAG code analyses, positions of these devices are irrelevant, and the fragmentation characteristics are assessed in reference to the fragment trajectory angles $\Theta^{\prime}$ calculated from the CALE code [2] cell velocities at the time of the shell breakup. Once the shell breaks up and fragments are formed, fragment velocities may change with time due to a number of reasons, including the air drag and the rigid body motion induced at the time of the shell break up. Assuming that the fragment trajectory angles $\Theta$ do not change with time (that is the rigid body motion and the lateral drift of fragments due to air resistance is relatively small) and that the definitions of angles $\Theta$ and $\Theta$ ' are approximately identical, the PAFRAG model enables prediction of crucial characteristics of explosive fragmenting munitions including the number of fragments, the fragment size distribution, and the average fragment velocities. 


\section{PAFRAG modelling}

Similarly to fragmentation arena test fragment sampling assumptions, the PAFRAG fragmentation model assumes that for any point within a fixed $\Theta$-angle zone the fragment number distribution $N_{j}(m)$ is uniform and independent of the altitude and the azimuth angles $\Theta$ and $\phi$, respectively. Hence, the total fragment number distribution is given by

$$
N(m)=\sum_{j}^{L} N_{j}(m)
$$

In equation (1) $m$ is the fragment mass, $L$ is the number of altitudinal $\Theta$-angle zones, $0 \leq \Theta \leq \pi$, and $N_{j}(m)$ is the fragment number distribution function for the $j$ th zone. For convenience, all $\Theta$-zones are assumed to have the same altitudinal lengths of $\Delta \Theta=\pi /(L-1)$, except for the first and the last "half-length" zones with lengths of $1 / 2 \Delta \Theta$. In the fragmentation ammunition arena testing practice adopted at the US Army Armament Research Development and Engineering Center, the number altitudinal zones is usually $L=37$, resulting in uniform $\Theta$-angle resolution of $\Delta \Theta=5^{\circ}$. Accordingly, the $\Theta$-zones are identified by the middle of the zone altitudinal angles $\Theta_{j}$ given by the following series

$$
\Theta_{j}= \begin{cases}1 / 4 \Delta \Theta, & j=1, \\ \Delta \Theta(j-1), & 2 \leq j \leq L-1, \\ \pi-1 / 4 \Delta \Theta, & j=L .\end{cases}
$$

In the case of traditional fragmentation arena testing, all individual fragment number distribution functions $N_{j}(m)$ for all polar $\Theta$-zones are determined directly from the test data. The main drawback of this approach is the extremely high testing costs limiting the fragmentation arena testing to final ammunition fragmentation characterization. Alternatively, the PAFRAG modeling and experimentation is a relatively low-cost procedure enabling accurate assessment of the fragmenting munition performance at the research, design, and development phases. In the PAFRAG approach the individual $\Theta$-zone fragment number distribution functions $N_{j}(m)$ are computed analytically from the sawdust or water tank fragment recovery test data, $N(m)$. Mathematically, the PAFRAG fragmentation modeling is a solution of the inverse problem of equation (1), i.e. determining a series of individual $N_{j}(m)$ 's for given $N(m)$. Since with PAFRAG approach, the $N(\mathrm{~m})$ function is assessed based on approximately $98-99 \%$ fragment recovery data, the accuracy of PAFRAG predictions is high.

Figure 1 shows results of high-strain high-strain-rate CALE modeling and flash radiographic images of a representative natural fragmentation warhead at 30 and $50 \mu \mathrm{s}$, and at 300 and $500 \mu \mathrm{s}$ after detonation. Upon initiation of the high explosive, rapid expansion of high-pressure high-velocity detonation products results in high-strain high-strain-rate dilation of the hardened fragmenting steel shell, which eventually ruptures generating a "spray" of high-velocity steel 
fragments. As shown in the model, the rear end of the warhead has a cylindrical cavity for the projectile tracer material. Following the expansion of the detonation products, the tracer holder fractures and the resulting fragments are projected in the negative direction of the $z$-axis, without contributing to the warhead lethality but posing potential danger to the gunner. As evidenced from the series of flash radiographic images shown in Figure 1, the tracer holder section of the warhead breaks up into a number of relatively large fragments that may cause serious or fatal injuries to the gunner.

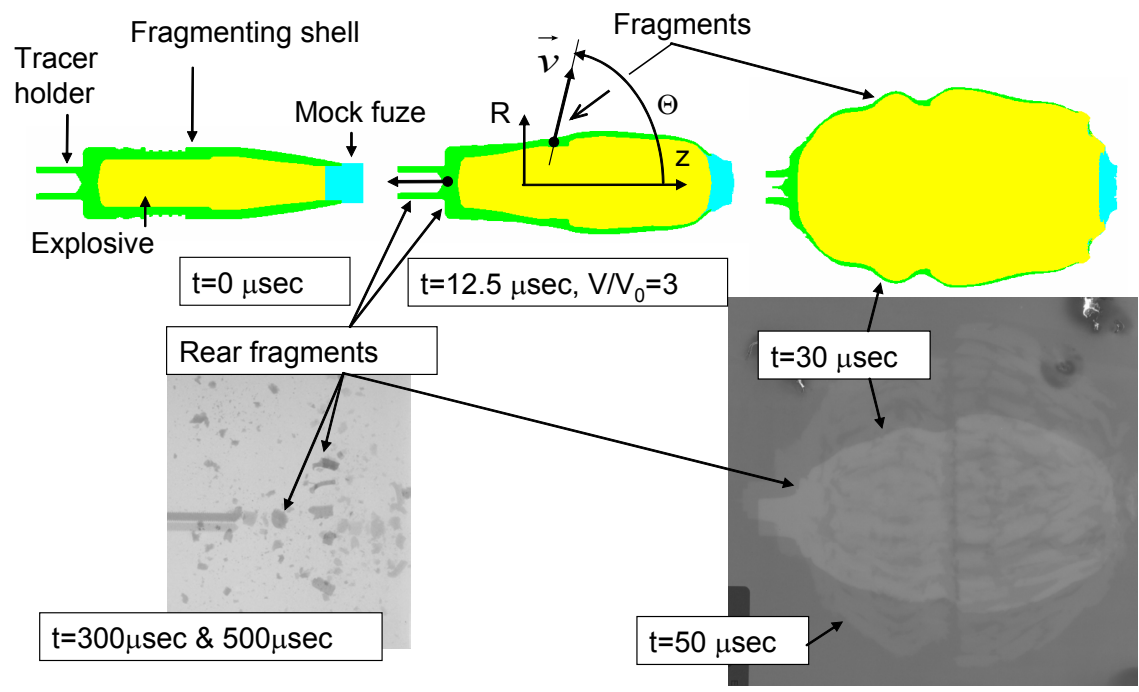

Figure 1: Results of CALE modeling and flash radiographic images of a natural fragmentation warhead at 30 and $50 \mu$ s (test No. X-969), and at 300 and $500 \mu$ s (test No. Y-070) after detonation.

CALE analyses had been conducted until approximately $30 \mu$ s after the charge initiation. As shown in the figure, CALE modeling results are in very good agreement with flash radiographic images of the fragmented warhead. The fundamental assumption of all fragmentation analyses presented in this work was that the fragmentation occurs simultaneously throughout the entire body of the shell. Following Pearson [3], the fragmentation of steel shells with the idealized cylindrical geometries occurs approximately at 3 volume expansions, the instant of fragmentation defined as the time at which the detonation products first appear emanating from the fractures in the shell. Accordingly, at approximately 3 volume expansions $(12.5 \mu \mathrm{s})$, the fragmenting steel shell was assumed completely fractured, and the CALE-code cell flow field data was passed to PAFRAG-MOTT and PAFRAG-FGS2 fragmentation modeling.

For a large part the PAFRAG-MOTT fragmentation model is based on the Mott's theory of break-up of cylindrical "ring-bombs" [4], in which the average length of the resulting circumferential fragments is a function of the radius and velocity of the ring at the moment of break-up, and the mechanical properties of 
the metal. Accordingly, in the PAFRAG-MOTT model the "random variations" in fragment sizes of natural fragmentation warheads are accounted through the following fragment distribution relationship

$$
N(m)=\sum_{j}^{L} N_{0 j} e^{-\left(m / \mu_{j}\right)^{1 / 2}} .
$$

In eqn. (3) $N_{0 j}$ and $\mu_{j}$ represent number of fragments and one half of the average fragment mass in the $j$-th $\Theta$-zone, respectively, computed from the CALE-code data.

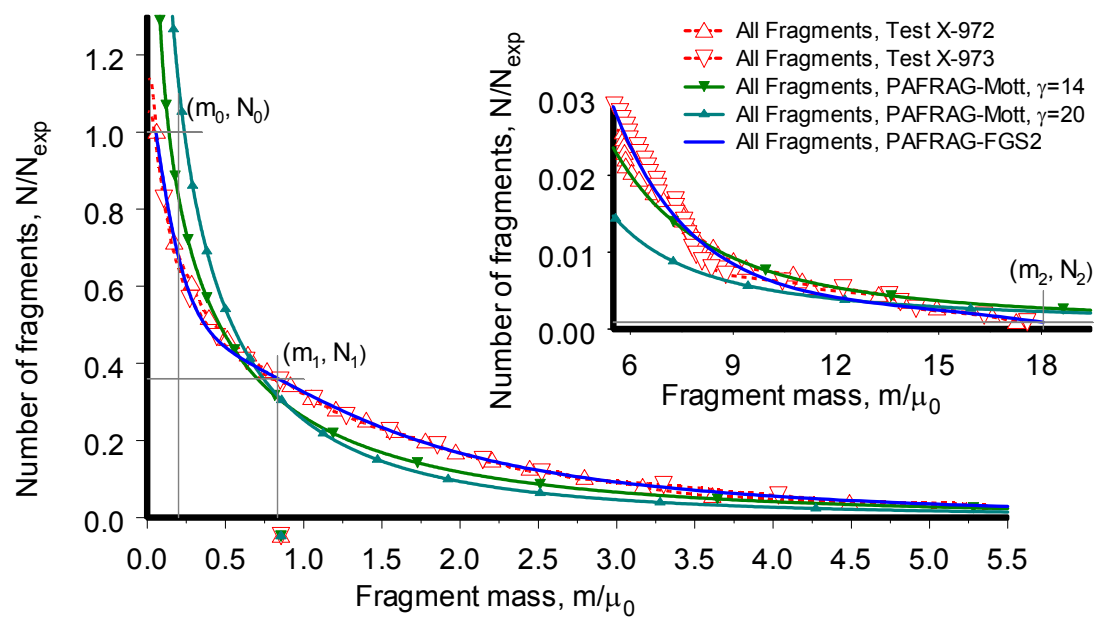

Figure 2: Cumulative number of fragments versus fragment mass, $N=N(m)$, for small-to-moderate weight $\left(m / \mu_{0}<5.5\right)$ and relatively large $\left(m / \mu_{0}>5.5\right)$ fragments.

The PAFRAG-FGS2 fragmentation model is defined in parametric form as

$$
\left[\begin{array}{c}
N_{k}\left(\xi_{k}\right) \\
m\left(\xi_{k}\right)
\end{array}\right]=\left[\begin{array}{c}
\sum_{j}^{L} \frac{m_{j}}{\sum_{j}^{L} m_{j}}\left(a_{N 0 k}+a_{N 1 k} \xi_{k}+a_{N 2 k} \xi_{k}^{2}+a_{N 3 k} \xi_{k}^{3}\right) \\
a_{m 0 k}+a_{m 1 k} \xi_{k}+a_{m 2 k} \xi_{k}^{2}+a_{m 3 k} \xi_{k}^{3}
\end{array}\right]
$$

In eqn. (4) $\xi_{k}$ is a non-dimensional parameter, $0 \leq \xi_{k} \leq 1, k$ is the curve index, $k=0,1$, and sixteen coefficients $a_{N}$ 's and $a_{m}$ 's are obtained by fitting two curve segments $k=0$ and $k=1$ with conditions of curve and tangent continuity at the adjacent ends.

Results of the PAFRAG modeling are given in figures 2 through 5. Figure 2 shows plots of the cumulative number of fragments versus fragment mass for 
small-to-moderate weight $\left(m / \mu_{0}<5.5\right)$ and for relatively large $\left(m / \mu_{0}>5.5\right)$ fragments calculated with PAFRAG-MOTT and PAFRAG-FGS2 models. As shown in the figure, attempting to fit the sawdust fragment recovery data with the PAFRAG-MOTT model by changing parameter $\gamma$ "rotated" the curve, but did not yield an accurate fit to the data. Accordingly, more a "flexible" PAFRAGFGS2 model was applied. As shown in the figure, using the PAFRAG-FGS2 model resulted in accurate fit throughout the entire range of data. Accordingly, PAFRAG-FGS2 model was used for all further analyses.

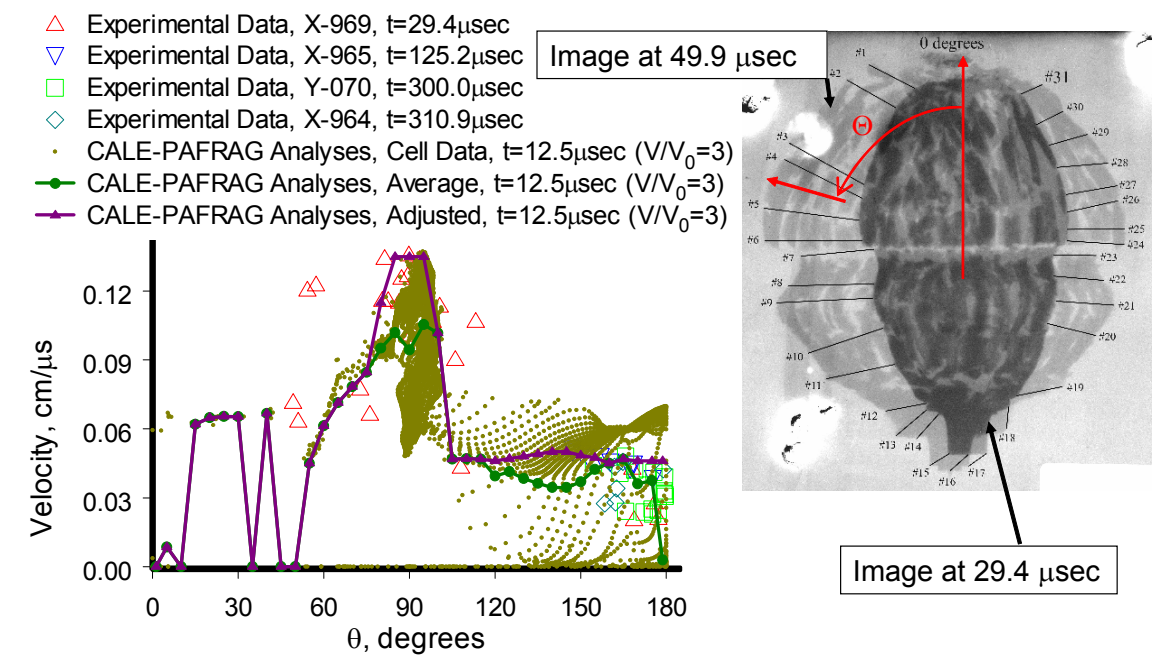

Figure 3: Fragment velocities versus fragment spray angle $\Theta$ and flash radiographic images at 29.4 and $49.9 \mu$ s (test No. X-969) after detonation.

Figure 3 shows the PAFRAG model fragment velocity predictions compared with the experimental data. The experimental values of fragment velocities of the main fragment spay $\left(80^{\circ} \leq \Theta \leq 100^{\circ}\right)$ were obtained from the flash radiographic images at 29.4 and $49.9 \mu \mathrm{s}$. Velocities of the rear fragments broken off from the tracer section of the shell (which move significantly slower than fragments from the main spray) were assessed from the flash radiographic images at 125.2, 300.0 and $310.9 \mu \mathrm{s}$. PAFRAG model prediction of the "average" $\Theta$-zone fragment velocities was obtained from the momentum averaged CALE-code flow field cell velocities. As shown in the figure, the agreement between the PAFRAG model fragment velocities predictions and the data is good.

Figure 4 shows PAFRAG model predictions of the fragment mass distribution versus the spray angle $\Theta$; the zonal fragment mass $m_{j}$ and the cumulative fragment mass $M$ distribution functions were computed from CALE-code cell flow field data. For representation clarity, the cumulative fragment mass function $M$ is defined in terms of angle $180^{\circ}-\Theta$, not the spray angle $\Theta$. As shown in the figure, the PAFRAG model prediction of the cumulative fragment mass 
distribution $M$ is in good agreement with the available experimental data at $\Theta=161.6^{\circ}$ (the Celotex ${ }^{\mathrm{TM}}$ and the water test fragment recovery) and at $\Theta=180^{\circ}$ (the sawdust fragment recovery).

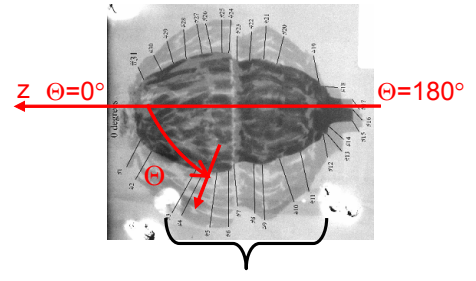

Lethal fragments

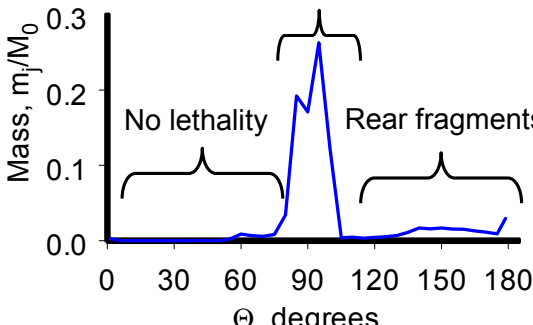

$\Theta$, degrees
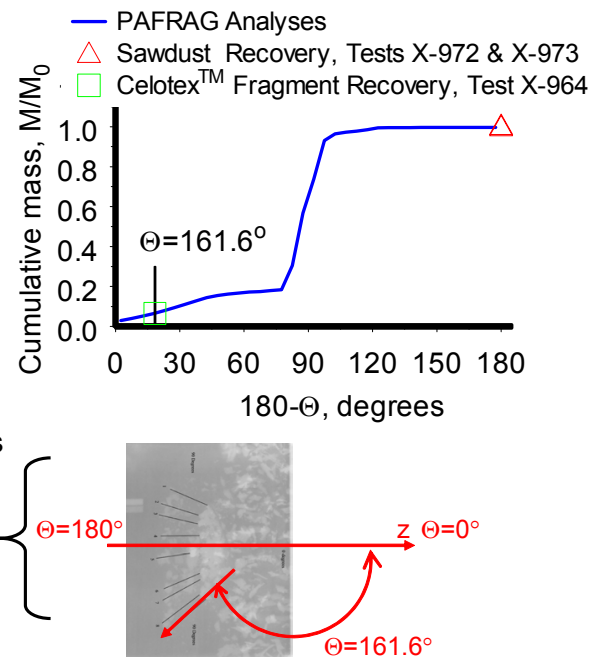

Figure 4: PAFRAG analyses of fragment mass distribution versus $\Theta$. Cumulative fragment mass distribution from PAFRAG analyses is in excellent agreement with experimental data.

As shown in figure 4, PAFRAG modeling predicts that the majority of the munition's fragment spray is projected into a relatively narrow $\Theta$-zone in the direction perpendicular to the projectile's axis, approximately at angles $80^{\circ} \leq \Theta \leq 100^{\circ}$. This is in good agreement with the flash radiography data showing no fragments projected to the projectile's anterior region, $0^{\circ} \leq \Theta \leq 50^{\circ}$. The fragment velocity "spikes" in the region of $0^{\circ} \leq \Theta \leq 50^{\circ}$ (see fragment velocity plot, Fig. 3), are due the numerical "noise" from a few "stray" mix-material computational cells from the CALE modeling. Because there is no considerable fragment mass in the front $\Theta$-zones, the overall effect of these errors is negligible, and the "average" fragment velocity in the $0^{\circ} \leq \Theta \leq 50^{\circ}$ region should be disregarded.

As evidenced from the flash radiographic images presented in Fig. 4, the tracer holder portion of the warhead breaks up into a number of relatively large fragments projected in the negative $z$-axis direction, back towards the gunner. As shown in Fig. 4, in excellent agreement with the Celotex ${ }^{\mathrm{TM}}$ and the water test fragment recovery data, PAFRAG modeling predicts that approximately $7.2 \%$ of the total fragment mass is projected to the "rear", in the region of $161.6^{\circ} \leq \Theta \leq 180^{\circ}$. Since according to PAFRAG modeling and the flash radiography data, Fig. 3, the velocities of these fragments is approximately 0.05 $\mathrm{cm} /$ microsecond, the broke-up pieces of the projectile's tracer holder are capable of causing serious injuries or death to the gunner. 
Figure 5 shows PAFRAG-FGS2 model predictions of the cumulative number of fragments versus fragment mass, $N=N(m)$, and of the $\Theta$-zonal number of fragments versus $\Theta, N_{j}=N_{j}(\Theta)$, for both the total "all fragments" and the "rear only" $\left(161.6^{\circ} \leq \Theta \leq 180^{\circ}\right)$ modeling cases. The "all fragments" fragment distribution was assessed from the sawdust fragment recovery tests that included fragments from the tracer section together with all fragments from the entire shell. The "rear only" fragment distribution was obtained from the Celotex" and from the water test fragment recovery experimentation and accounted only for fragments projected at angles greater than approximately $161.6^{\circ}$. The limiting rear fragment collection angle of $\Theta=161.6^{\circ}$ represents the altitudinal angle $\Theta$ covering the fragment recovery surface area.

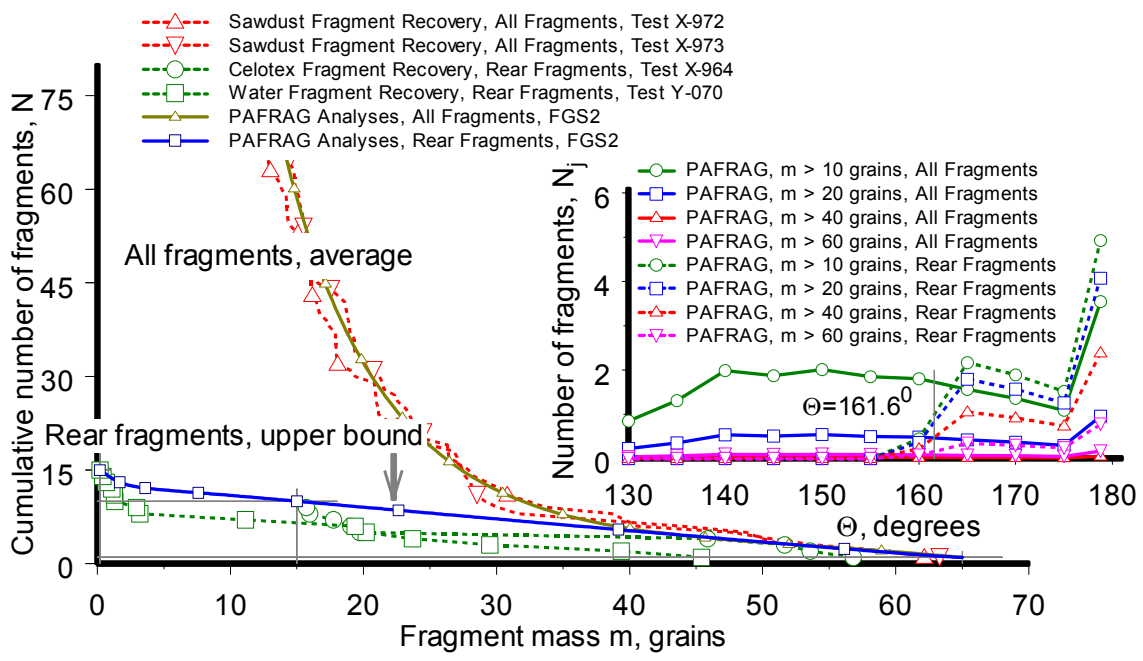

Figure 5: Cumulative number of fragments versus fragment mass and number of fragments versus $\Theta$, for total "all fragments" and "rear only" $\left(\Theta>161.6^{\circ}\right)$ distributions.

As shown in Fig. 5, the "rear fragments" PAFRAG-FGS2 model fragment distribution was obtained by fitting eqn. (3) to the upper bound of the Celotex ${ }^{\mathrm{TM}}$ and water test recovery data, providing an additional "safety" margin for the safe separation distance analyses. Since in a typical fragmentation warhead only a few fragments are projected backward towards the gunner, establishing a statistically robust database from the conventional fragmentation arena test requires repeated experimentation and is expensive. In contrast, the data from the PAFRAG modeling offers to munition designers more warhead performance information for significantly less money spent. The PAFRAG provides more detailed and more statistically accurate warhead fragmentation data for ammunition safe separation distance analysis, as compared to the traditional fragmentation arena testing approach. 


\section{PAFRAG model assessment of lethality and safety separation distance}

The safety separation distance analyses presented in this work were performed employing the JMEM/OSU Lethal Area Safety Program for Full Spray Fragmenting Munitions code [6] and the Wedge model computational module. According to [7], the safe separation distance is defined as fixed distance from the weapon's launch platform and personnel beyond which functioning of the munition presents an acceptable risk of a hazard to the personnel and the platform. Accordingly, the safe separation hazard probability had been calculated based on the warhead's fragment spray ability to strike and to penetrate exposed (bare) skin tissue of unprotected gun crew personnel. According to [7], the maximum total risk to the munition crew at safe separation distance is generally accepted as $10^{-6}$.

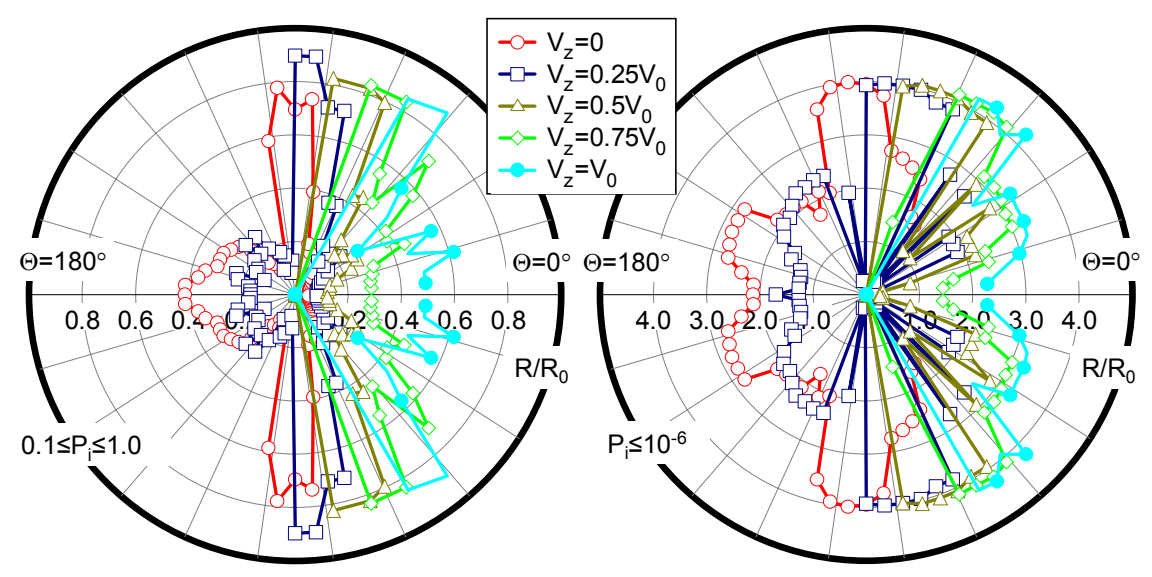

Figure 6: Areas of $0.1 \leq P_{i} \leq 1$ and $P_{i} \leq 10^{-6}$ unprotected personnel risk hazards for varying projectile lunch velocities.

The input for the lethality and safe separation distance analyses included a range of possible ballistic projectile trajectories and the static PAFRAG FGS2 model predictions of the fragment spray blast characteristics. Figure 6 shows resulting plots of areas with $0.1 \leq P_{i} \leq 1$ and $P_{i} \leq 10^{-6}$ unprotected personnel risk hazards for varying projectile lunch velocities. As shown in Fig. 6, the projectile launch velocity has a significant effect on both the munition lethality $\left(0.1 \leq P_{i} \leq 1\right)$ and the safety $\left(P_{i} \leq 10^{-6}\right)$. As shown in the figure, if the gun operates normally and launches the projectile with the nominal muzzle velocity of $V_{0}$, all fragments are projected in the forward direction, posing no danger to the gun crew. However, if the gun misfires $\left(V_{z}<<V_{0}\right)$ and the munition is detonated, the results may be catastrophic. 


\section{Acknowledgements}

Messrs. K. P. Ko of PM MAS and A. N. Cohen of US Army RDEC-ARDEC are acknowledged for providing funds that made this work possible. Mr. K. W. Ng of US Army RDEC-ARDEC and Mrs. Y. Wu of SAIC are acknowledged for their contribution in CALE modeling. Mr. W. J. Poulos of US Army RDECARDEC is acknowledged for performing experiments. Mr. T. Fargus and Mrs. D. L. Snyder of US Army RDEC-ARDEC are acknowledged for performing lethality and safety separation distance analyses.

\section{References}

[1] Joint Munition Effectiveness Manual. "Testing and data reduction procedures for high-explosive munitions", Report FM 101-51-3, Revision 2, 8 May 1989.

[2] Tipton R. E., "CALE users manual”, Version 910201, Lawrence Livermore National Laboratory, 1991

[3] J. Pearson, "A fragmentation model for cylindrical warheads", Technical Report NWC TP 7124, Naval Weapons Center, China Lake, California, December 1990.

[4] N. F. Mott, F.R.S., "Fragmentation of steel cases", Proc. Roy. Soc., Vol. 189, pp. 300-308, 1947.

[5] J. C. Ferguson, "Multivariable curve interpolation", Report No. D222504, The Boing Co., Seattle, Washington, 1963.

[6] Joint Technical Coordination Group for Munitions Effectiveness. "Computer program for general full spray materiel MAE computations", Report 61 JTCG/ME-70-6-1, 20 December 1976, Change1: 1 April 1991.

[7] "Guidance for Army Fuze Safety Review Board safety Characterization", US Army Fuze Office, January, 1999. 